\title{
Distances between Rats in Reverberation Chambers Used for Large-Scale Experiments
}

\author{
Sangbong Jeon ${ }^{1} \cdot$ Wook Jang ${ }^{2} \cdot$ Ae-Kyoung Lee ${ }^{1} \cdot$ Hyung-Do Choi $^{1} \cdot$ Jeong-Ki Pack $^{3}$. \\ Jianqing Wang ${ }^{4}$ Dongho Kim ${ }^{2, *}$
}

\begin{abstract}
We investigate the whole-body average specific absorption rate (WBA-SAR) of rats under various plane wave exposure characteristics, including different polarizations, incidence angles, distances between rats, and total number of rats. Unlike many other studies, we start our SAR analysis from one rat and expand it to 27 rats facing random directions in a three-dimensional area. In a one-rat analysis, we examine how the incidence direction and polarization affect the SAR of a single rat. Moreover, we look into how various incidence polarizations behave differently after they are transmited through a rat, the information of which is then used to analyze the effect of spacing among 27 randomly arranged rats. Next, we analyze the effect on spacing of the 27 rats deployed under a 52-plane-wave exposure, which is introduced to mimic a realistic reverberation chamber $(\mathrm{RC})$ environment. We show the deviation in WBA-SAR according to the distance between rats, which provides guidelines for selecting the appropriate rat distance based on the number of animals and the exposure deviation within a limited working volume in an $\mathrm{RC}$ for large-scale experiments.
\end{abstract}

Key Words: Incident Angle, Incident Polarization, Rat Distance, SAR, Standard Deviation, Statistical Analysis.

\section{INTRODUCTION}

Various types of experimental systems have been used to study the toxicology and carcinogenesis of animal exposure to electromagnetic waves [1]. A reverberation chamber (RC) is one of the most widely used facilities, which not only provides a statistically uniform field distribution within a specific volume, but also permits the animals to move freely around in their cages [2]. Although an $\mathrm{RC}$ offers a uniform distribution, obtaining an evenly distributed specific absorption rate (SAR) is another important aspect that should be accurately analyzed for individual animals within an RC. In [3], it was reported that the SAR de- pends on certain parameters such as the polarization or rat arrangement, which was investigated in an RC. In [4], using a rat phantom in an $\mathrm{RC}$, the authors showed that a two-dimensional statistical SAR distribution can explain the corresponding distance between rats. However, despite these previous studies, there are still no practical guidelines for an optimum cage deployment in a practical RC design, which should consider the overall effect of the incident polarization, angle, distance between rats, and arrangement.

In this letter, we provide a new method for determining the optimum distance between rats to obtain even SAR levels among rats; this will be used in the RC design for a preliminary

\footnotetext{
Manuscript received September 7, 2020 ; Revised December 4, 2020 ; Accepted February 14, 2021. (ID No. 20200907-136J)

${ }^{1}$ Radio \& Satellite Research Division, Electronics and Telecommunications Research Institute (ETRI), Daejeon, Korea.

${ }^{2}$ Department of Electrical Engineering, Sejong University, Seoul, Korea.

${ }^{3}$ Radio Sciences \& Engineering Department, Chungnam National University, Daejeon, Korea.

${ }^{4}$ Electrical and Electronic Engineering, Nagoya Institute of Technology, Nagoya, Aichi, Japan.

"Corresponding Author: Dongho Kim (e-mail: dongkim@sejong.ac.kr)
}

This is an Open-Access article distributed under the terms of the Creative Commons Attribution Non-Commercial License (http://creativecommons.org/licenses/by-nc/4.0) which permits unrestricted non-commercial use, distribution, and reproduction in any medium, provided the original work is properly cited.

(c) Copyright The Korean Institute of Electromagnetic Engineering and Science. All Rights Reserved. 
study promoted by a Korea - Japan joint animal study. Specifically, we analyze how the incident polarizations and angles affect the SAR of a single rat, which will then be expanded to a threedimensional (3D) random arrangement of 27 rats to examine the rat-distance effect on the SAR variations.

\section{METHODS}

The overall simulation setup used throughout this work is as follows:

- Simulation program and data: Sim4Life (Zurich MedTech AG) [5]

- Rat model: big male rat model with a straight tail (IT'IS Foundations) (Sprague Dawley, $567 \mathrm{~g}$ )

- Incident wave: plane wave with a $1 \mathrm{~V} / \mathrm{m}$ electric field at 900 $\mathrm{MHz}$

- Analysis data: whole-body average SAR (WBA-SAR, $\mu \mathrm{W} / \mathrm{kg}$ )

The big male rat model used is shown in Fig. 1(a), where each face of the imaginary rectangular parallelepiped is touching the outermost body part of the rat. The distances $(d)$ between neighboring rats are the same along the $\mathrm{x}^{-}, \mathrm{y}^{-}$, and $\mathrm{z}$-axes, as shown in Fig. 1(b), and will be used for the 27-rat arrangement.

\section{RESULTS}

Fig. 2 shows how the incident angles and polarization of a plane

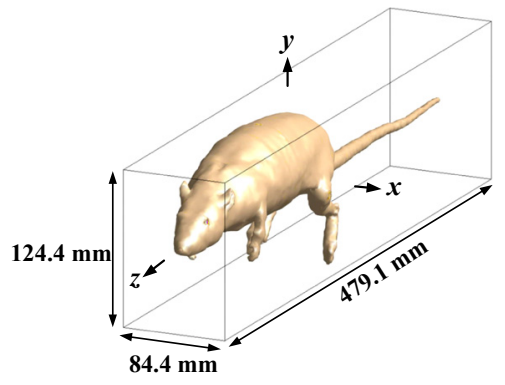

(a)

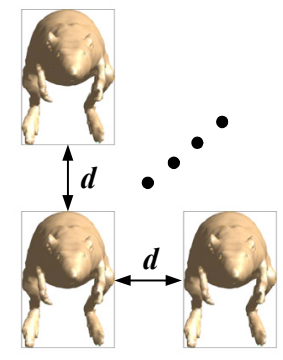

(b)
Fig. 1. (a) Big male rat model and (b) rat distance for 27-rat arrangement shown in Fig. 4.
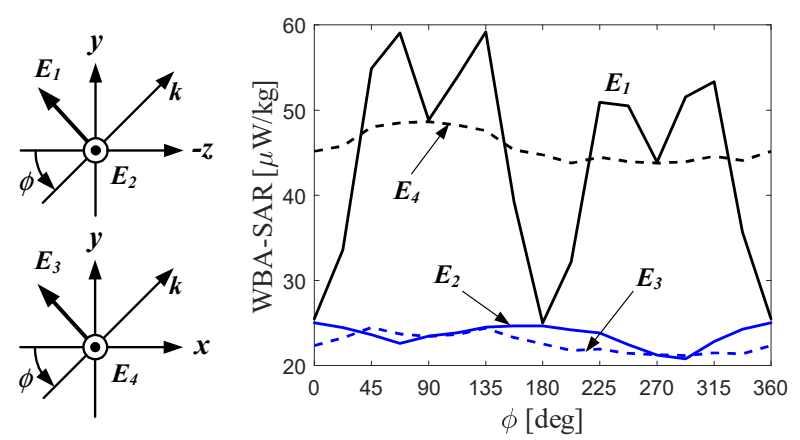

Fig. 2. WBA-SAR variations of a single rat for different incident angles and polarizations. wave affect the WBA-SAR of a single rat. We can see that, when the incident polarization is parallel to the rat axis ( $z$-axis), and the incident angle is facing the maximum cross-section area of the rat, the WBA-SAR increases (see $E_{1}$ and $E_{4}$ ). On the contrary, for the perpendicular incidence $\left(E_{2}\right.$ and $\left.E_{3}\right)$, the WBA-SAR is very low.

We examine how the incident polarization perturbs the electric fields around the rat, as shown in Fig. 3 for two specific cases of $E_{3}$ and $E_{4}$ under $\Phi=0^{\circ}$. In both cases, there is strong interference between the incident and scattered waves in region $A$, which produces high periodic oscillations. In region B, however, the scattering properties are extremely different from each other, and a rat under a $z$-polarized incidence induces a high shadowing effect immediately behind the rat. In contrast, for $y$ polarized incidence, the total $\mathrm{E}$ field is immediately restored to the incident level. Therefore, when we arrange a large number of rats in a particular area such as in an $\mathrm{RC}$, we can expect that the SAR of each rat will be significantly affected by the incident polarization, direction, distance between rats, and the direction the rat is facing.

To analyze the overall effect of these parameters, we arranged 27 rats as shown in Fig. 4. For the arrangement, we first arranged

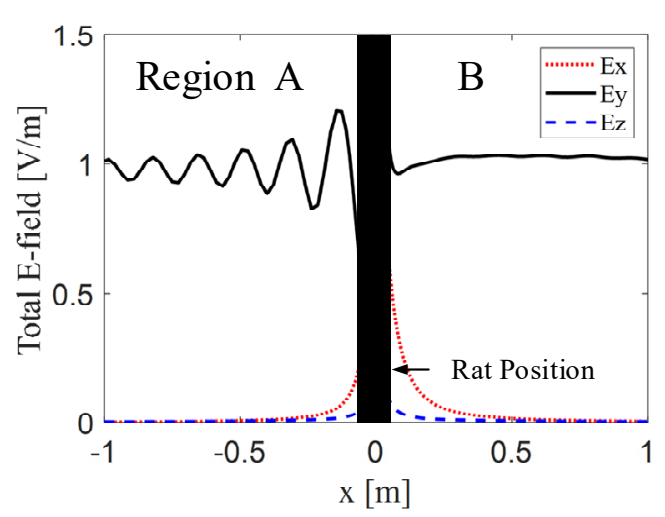

(a)

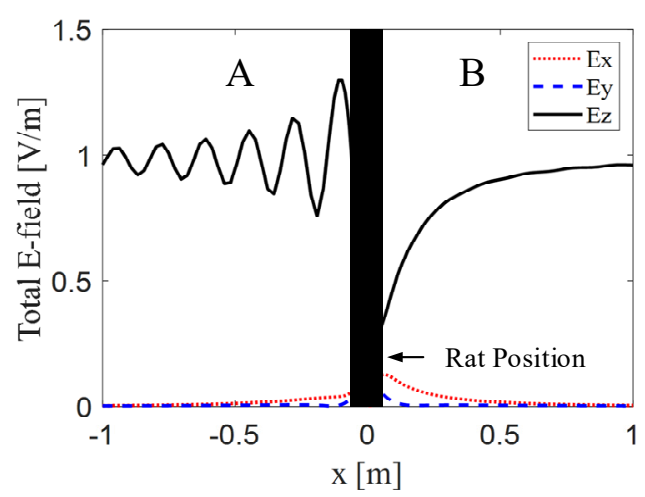

(b)

Fig. 3. Magnitude of total E-field behaviors for (a) $y$-polarized $\left(\mathrm{E} 3, \Phi=0^{\circ}\right)$ and (b) $z$-polarized incident $\left(\mathrm{E} 4, \Phi=0^{\circ}\right)$ waves propagating toward the positive $x$-axis. 


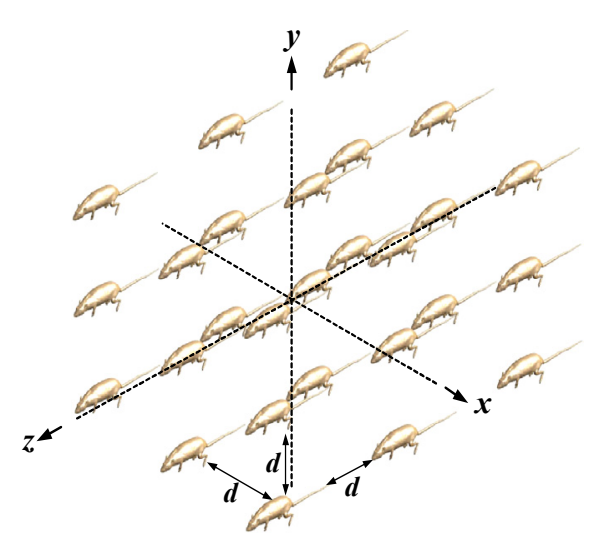

(a)

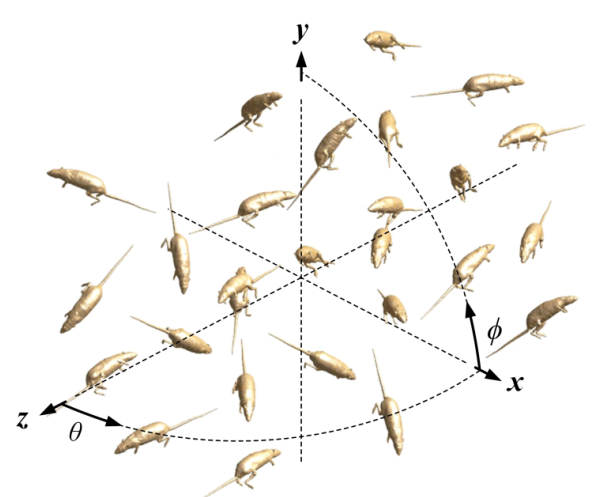

(b)

Fig. 4. Arrangement of 27 rats for (a) regular directions and (b) facing random directions according to the random rotation angles defined in Table 1.

all rats regularly in a $3 \times 3 \times 3$ formation with the same distance $(d)$ between rats. We then rotated each rat according to the random angles given in Table 1 , where the center of rotation of each rat is at the center of each rectangular parallelepiped. All rotation angles were chosen from uniformly distributed random numbers using MATLAB such that none of the rats overlapped even at the minimum distance of $d=50 \mathrm{~mm}$. A regular deployment, as shown in Fig. 4(a), is unrealistic considering the animals' movement. However, the deployment of Fig. 4(b) is more realistic for a simulation. As shown in Fig. 5, the average WBA-SAR and the corresponding standard deviation (SD) of the 27 rats were analyzed for both regular and random deployments. Here, we used 52 incident plane waves coming from every $45^{\circ}$ angle to mimic the environment in the RC. As the distance between rats increased in both deployments, the average WBA-SAR reached the WBA-SAR of one rat at $d=400$ $\mathrm{mm}$ because the shadowing effect gradually diminished for a larger spacing. In addition, note that there is a small difference between the average WBA-SARs of the regular and random deployments.

In this study, the same spacing $d$ was used for the $\mathrm{x}^{-}, \mathrm{y}^{-}$, and $\mathrm{z}$-axes for a simple arrangement and calculation. However, in an actual experimental environment, in many cases, it is inevitable
Table 1. Random rotation angles of each rat for the arrangement of Fig. 4(b)

\begin{tabular}{cccccc}
\hline Rat no. & $\theta\left(^{\circ}\right)$ & $\phi\left(^{\circ}\right)$ & Rat no. & $\theta\left(^{\circ}\right)$ & $\phi\left(^{\circ}\right)$ \\
\hline 1 & 62 & 169 & 15 & 60 & 19 \\
2 & 65 & 315 & 16 & 138 & 159 \\
3 & 15 & 30 & 17 & 135 & 300 \\
4 & 32 & 269 & 18 & 67 & 323 \\
5 & 140 & 290 & 19 & 32 & 70 \\
6 & 50 & 345 & 20 & 150 & 0 \\
7 & 162 & 66 & 21 & 10 & 110 \\
8 & 21 & 215 & 22 & 134 & 190 \\
9 & 178 & 108 & 23 & 60 & 60 \\
10 & 97 & 48 & 24 & 31 & 359 \\
11 & 127 & 76 & 25 & 37 & 0 \\
12 & 150 & 250 & 26 & 163 & 107 \\
13 & 155 & 25 & 27 & 122 & 50 \\
14 & 40 & 60 & - & - & - \\
\hline
\end{tabular}

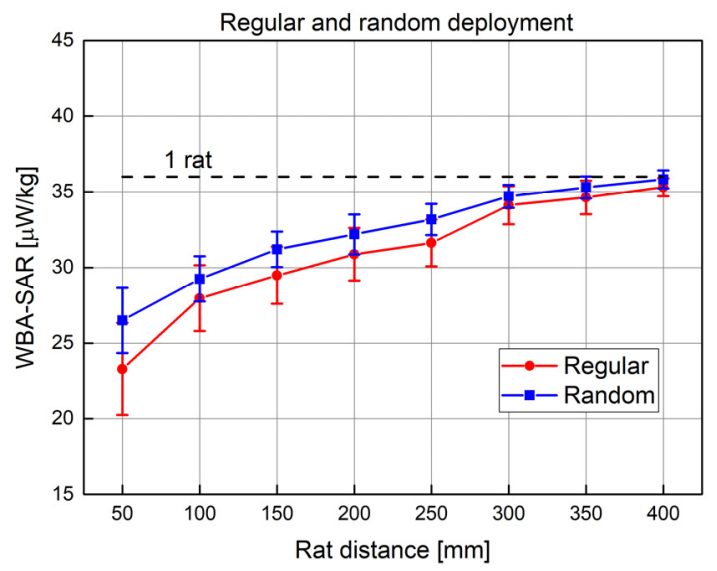

Fig. 5. Average WBA-SAR of 27 rats.

for different intervals to be used depending on the axis. The working volume of the RC designed and manufactured for the Korea-Japan joint animal study was $1.5 \times 1.3 \times 1.5 \mathrm{~m}^{3}$, and 75 rats (one per cage) had to be placed within this limited space. The final distances between cages were $150 \mathrm{~mm}, 50 \mathrm{~mm}$, and $180 \mathrm{~mm}$ in the three directions, respectively. The SAR calculations were conducted, and the results for the 27 regular and random arrangements were $30.42 \pm 1.4 \mu \mathrm{W} / \mathrm{kg}$ and $31.23 \pm$ $1.4 \mu \mathrm{W} / \mathrm{kg}$, respectively. The average WBA-SAR was $0.6 \mathrm{~dB}$ lower than the single rat case, and the SAR value of each rat falls within $\pm 5 \%$ of the average WBA-SAR of the 27 rats. In addition, we also found that the average WBA-SARs between regular and random deployments was no greater than $2.6 \%$.

\section{CONCLUSION}

In large-scale SAR experiments within a limited working 
volume of an $\mathrm{RC}$, as the distance between objects (rats) increases, the shadowing effect decreases, but requires a larger space. To provide the relation between the rat distance and exposure deviation (mean and SD), 27 rats in an RC were simulated according to their deployments, which were drawn from a statisti$\mathrm{cal}$ analysis of their $3 \mathrm{D}$ arrangement. Using our results, we will reflect the distance of $150 \times 50 \times 180 \mathrm{~mm}^{3}$ between the cages in the RC design for the Korea-Japan joint animal study. In the future, we will carry out rat weight-based exposure simulations for 75 rats under the designed distance between rats. In addition, we expect that the proposed approach will be a helpful guideline for a practical RC design for large-scale experiments.

This work was supported by the ICT R\&D program of MSIP/IITP (No. 2019-0-00102, A Study on Public Health and Safety in a Complex EMF Environment).

\section{REFERENCES}

[1] A. Paffi, C. Merla, R. Pinto, G. A. Lovisolo, M. Liberti, C.

Sang Bong Jeon

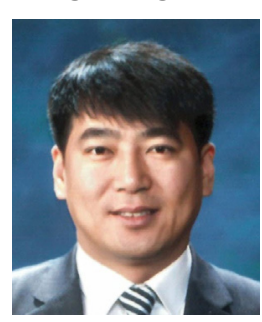

received his B.S., M.S., and Ph.D. degrees in electronic engineering from Yeungnam University, Gyeongsan, Korea, in 2001, 2003, and 2007, respectively. From 2008 to 2010, he was a senior research engineer at the Korea Radio Promotion Association, Seoul, Korea, where he conducted research in the fields of electromagnetic compatibility technology. Since 2010, he has been with Radio \& Satellite Research Division, Electronics and Telecommunications Research Institute, Daejeon, Korea. His research interests include bioelectromagnetics and electromagnetic compatibility.

Wook Jang

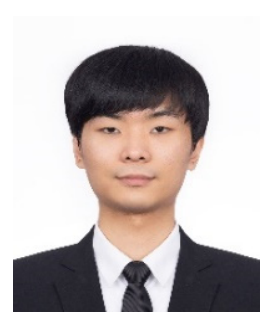

received the B.S. degree in electronics and information engineering from Sejong University, Seoul, Korea, in 2020. Since March 2021, he is currently working towards his M.S. degree at the electronic engineering, Sejong University. His research interests include the analysis and design of high-gain beam scanning antennas by using a partially reflective surface.
Marino, M. Repacholi, and F. Apollonio, "Microwave exposure systems for in vivo biological experiments: a systematic review," IEEE Transactions on Microwave Theory and Techniques, vol. 61, no. 5, pp. 1980-1993, 2013.

[2] K. B. Jung, T. H. Kim, J. L. Kim, H. J. Doh, Y. C. Chung, J. H. Choi, and J. K. Pack, "Development and validation of reverberation chamber type whole-body exposure system for mobile-phone frequency," Electromagnetic Biology and Medicine, vol. 27, no. 1, pp. 73-82, 2008.

[3] B. J. Klauenberg and D. Miklavcic, Radio Frequency Radiation Dosimetry and Its Relationship to the Biological Effects of Electromagnetic Fields. Dordrecht: Kluwer Academic Publishers, 2000.

[4] J. Chakarothai, J. Wang, O. Fujiwara, K. Wake, and S. Watanabe, "Dosimetry of a reverberation chamber for whole-body exposure of small animals, "IEEE Transactions on Microwave Theory and Techniques, vol. 61, no. 9, pp. 3435-3445, 2013.

[5] Zurich MedTech (ZMT), "Sim4Life 5.2," 2019; https:// www.zmt.swiss.
Ae-Kyoung Lee

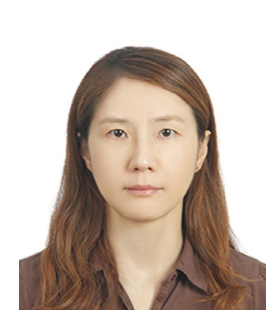

received her B.S. and M.S. degrees in electronics and engineering from Chungang University, Seoul, Korea in 1990 and 1992, respectively, and a $\mathrm{PhD}$ in radio science and engineering from Chungnam $\mathrm{Na}$ tional University, Daejeon, Korea in 2003. In 1992, she joined the Radio Technology Group at the Electronics and Telecommunications Research Institute, Daejeon, Korea, where she has been involved in projects on measurement technologies and numerical analyses of electromagnetic compatibility and human exposure to RF fields. Dr. Lee was the recipient of the Japan Microwave Prize at the 1998 Asia-Pacific Microwave Conference, Japan and the Technology Award from the Korea Electromagnetic Engineering Society in 1999.

\section{Hyung-Do Choi}

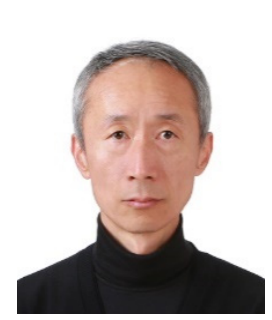

received his M.S. and Ph.D. degree in material sciences from Korea University in 1989 and 1996, respectively. Since 1997, he has been with Electronics and Telecommunications Research Institute, Daejeon, Korea, where he is presently a principal member of Radio \& Satellite Research Division. He has conducted research on the biological effects of RF radiation and has developed RF radiation protection standards. His current research interests include spectrum management, microwave tomography, and EMC countermeasures. 


\section{Jeong-Ki Pack}

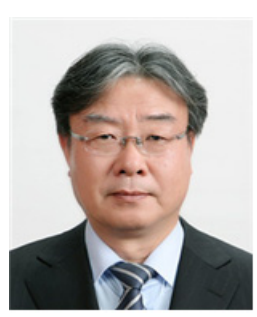

received his B.S. in Electronic Engineering from Seoul National University, Seoul, Korea, in 1978. From 1978 to 1983, we worked for the Agency for Defence Development in Korea as a researcher. He received his M.S. and Ph.D. degrees in 1985 and in 1988, respectively, in electromagnetic wave propagation from Virginia Tech., USA. He joined ETRI in 1988 and moved to Dong-A University in 1989. Since February 1995, he has been with Chungnam National University, Daejeon, Korea, as a professor at the Department of Radio Science and Engineering. He is currently an emeritus professor at the same department. His research interests include electromagnetic wave propagation and bioelectromagnetics.

\section{Jianqing Wang}

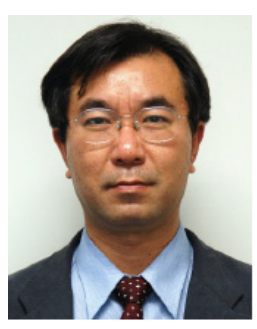

received the B.E degree in electronic engineering from Beijing Institute of Technology, Beijing, China, in 1984, and the M.E. and D.E. degrees in electrical and communication engineering from Tohoku University, Sendai, Japan, in 1988 and 1991, respectively. He was a Research Associate with Tohoku University and a Senior Engineer with Sophia Systems Co., Ltd. In 1997, he joined the Nagoya Institute of Technology, Nagoya, Japan, where he has been a Professor, since 2005. He has authored Body Area Communications (Wiley-IEEE), in 2012, and received the IEEE EMCS Technical Achievement Award, in 2019. He is IEEE Fellow. His current research interests include biomedical communications and electromagnetic compatibility.

\section{Dongho Kim}

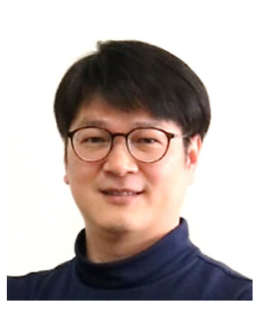

received the B.S. and M.S. degrees in electronics engineering from Kyungpook National University, Daegu, Korea, in 1998 and 2000, respectively, and the Ph.D. degree in electrical and electronics engineering from Korea Advanced Institute of Science and Technology (KAIST), Daejeon, Korea, in 2006. From 2000 to 2011, he was a Senior Researcher in Electronics and Telecommunications Research Institute (ETRI), Daejeon, Korea. In 2011, he joined the Department of Electrical Engineering, Sejong University, Seoul, Korea, where he is now a Professor. Prof. Kim is a member of the Institute of Electrical and Electronics Engineers (IEEE), an expert of the International Electrotechnical Commission (IEC), and a life-member of the Korean Institute of Electromagnetic Engineering and Science (KIEES). His research interests include advanced electromagnetic wave theory, antenna design, metamaterials, and bio-electromagnetics. 